\title{
Role of many-body entanglement in decoherence processes
}

\author{
Helen McAneney, ${ }^{1}$ Jinhyoung Lee, ${ }^{1}$ and M. S. Kim ${ }^{1}$ \\ ${ }^{1}$ School of Mathematics and Physics, The Queen's University, Belfast BT'7 1NN, United Kingdom
}

(Dated: February 9, 2020)

\begin{abstract}
A pure state decoheres into a mixed state as it entangles with an environment. When an entangled two-mode system is embedded in a thermal environment, however, each mode may not be entangled with its environment by their simple linear interaction. We consider an exactly solvable model to study the dynamics of a total system, which is composed of an entangled two-mode system and a thermal environment, and also an array of infinite beam splitters. It is shown that many-body entanglement of the system and the environment plays a crucial role in the process of disentangling the system.
\end{abstract}

PACS numbers: PACS number(s);42.50.Dv, 03.65.Bz

Decoherence has been studied in the context of quantum-classical correspondence, providing a quantumto-classical transition of a system [1]. A single-mode pure state becomes mixed and loses its quantum nature by decoherence in an environment. Although the dynamics of the system has been studied extensively, there has not been a thorough investigation on the quantum correlation between the system and the environment, which is behind the dynamics of the system. The decoherence process can be understood as a process of entanglement between the system and its environment which is composed of a many (normally, infinite) number of independent modes. The increase of the system entropy [2] may be due to the system-environment entanglement.

Most of the studies on decoherence have focused on a single-mode or single-particle system [3]. This is because if a many-body pure system is initially separable, its decoherence process is a straightforward extension of a single-body system. However, if there is entanglement in the initial pure system, the decoherence mechanism can be of a different nature. For an entangled two-mode pure system, each mode is generically in a mixed state and its passive linear interaction with an environment, which is normally in a mixed state, does not seem to bring about entanglement. What kind of correlation then causes the loss of entanglement initially in the system? In this paper, we answer this question by studying the quantum correlation of a two-mode entangled continuous-variable system with an environment in thermal equilibrium.

A continuous-variable state is defined in an infinitedimensional Hilbert space and it is convenient to study such a state using its quasiprobability Wigner function [4], $W(\tilde{\mathbf{x}})$, in phase space. For an $N$-mode field, the coordinates of phase space are composed of quadrature variables, $\tilde{\mathbf{x}}=\left\{q_{1}, p_{1}, \cdots, q_{N}, p_{N}\right\}$. Throughout the paper a vector is denoted in bold face and an operator by a hat. A fiber or a free space, through which a light field propagates, is normally considered a thermal environment. The dynamics of the field mode coupled to the thermal environment is, in the Born-Markov approximation, governed by the Fokker-Planck equation [4],

$$
\frac{\partial W(\tilde{\mathbf{x}})}{\partial \tau}=\frac{\gamma}{2} \sum_{i=1}^{2 N}\left(\frac{\partial}{\partial \tilde{x}_{i}} \tilde{x}_{i}+\frac{\tilde{n}}{4} \frac{\partial^{2}}{\partial \tilde{x}_{i}^{2}}\right) W(\tilde{\mathbf{x}})
$$

where $\gamma$ is the energy decay rate of the system and $\tilde{n}=$ $2 \bar{n}+1$ with $\bar{n}=\left[\exp \left(\hbar \omega / k_{B} T\right)-1\right]^{-1}$ is the average number of thermal photons at temperature $T . k_{B}$ is the Boltzmann constant.

It was shown by one of us that a single-mode Gaussian field interacting with a thermal environment can be modeled by the field passing through an array of infinite beam splitters [5]. A beam splitter is a simple passive linear device which keeps the Gaussian nature of an input field. Each beam splitter has two input ports. As the signal field is injected into one input port, it allows a degree of freedom for the other port where noise is injected. The collection of such degrees of freedom forms the environment. We assume a homogeneous thermal environment of temperature $T$ with all noise modes having the same physical properties. In Ref. [5], the Fokker-Planck equation (1) for a single-mode field was derived using the beam splitter. The model was used to study the dynamics of entanglement between a single-mode field and its environment [6].

Using the Fokker-Planck equation (11), one may study the dynamics of the system. However, it is hard to know the quantum correlation between the system and the environment as Eq. (11) is obtained by tracing over all environmental variables. In this paper, instead of tracing over all the environmental modes, we keep them to study the dynamics of entanglement of the system and the environment. A two-mode squeezed state, which may be generated by a nondegenerated optical parametric amplifier, is the most renowned and experimentally-relevant entangled state for continuous variables [7]. Its degree of entanglement increases as the degree of squeezing $s$ increases [8] and it becomes a regularized Einstein-Podolsky-Rosen state when $s \rightarrow \infty$ [9. In order to simplify the problem, we assume that only the mode $a_{2}$ of the system modes interacts with the environment while the other mode $a_{1}$ is isolated from it.

We consider an exactly solvable model of a two-mode 
system interacting with a homogeneous thermal environment, which results in the Fokker-Planck equation (1). For the Born-Markov approximation, we employ a timedependent coupling constant in the model. Let us start with a finite number $N$ of environmental modes interacting with the system. The interaction Hamiltonian, in the interaction picture, is

$$
\hat{H}_{I}(t)=\sum_{m=0}^{N-1} i \lambda_{N}(t)\left(\hat{a}_{2} \hat{b}_{m}^{\dagger}-\hat{a}_{2}^{\dagger} \hat{b}_{m}\right),
$$

where $\hat{b}_{m}$ is the bosonic annihilation operator for environmental mode $b_{m}$ and the coupling constant $\lambda_{N}(t)$ is determined so as to reproduce the Fokker-Planck equation (1).

It is convenient to introduce collective modes $c_{n}$ which are conjugate to $b_{m}$ under the Fourier transformation such that

$$
\hat{c}_{n} \equiv \sqrt{\frac{2}{N}} \sum_{m=0}^{N-1} \cos \left(\frac{2 \pi}{N} n m\right) \hat{b}_{m},
$$

where $\hat{c}_{n}$ is an annihilation operator for a collective mode $c_{n}$. The collective modes are related with the entangling nature of the modes $b_{m}$, for example, the quantum that $\hat{c}_{n}^{\dagger}$ creates from a vacuum is in an entangled state of $b_{m}$ modes.

The collective modes satisfy the boson commutation relation, $\left[\hat{c}_{n}, \hat{c}_{n^{\prime}}^{\dagger}\right]=\delta_{n n^{\prime}}$ and carry physical properties as bosonic modes. Using the collective mode, a state $\hat{\rho}$ is described by the characteristic function

$$
\chi_{c}(\mathbf{X})=\operatorname{Tr} \hat{\rho} \exp \left[i \mathbf{X} \cdot \hat{\mathbf{X}}^{T}\right],
$$

where $\hat{\mathbf{X}}=\left(\hat{Q}_{0}, \hat{P}_{0}, \hat{Q}_{1}, \hat{P}_{1}, \ldots, \hat{Q}_{N-1}, \hat{P}_{N-1}\right)$ with $\hat{Q}_{n}=$ $\left(\hat{c}_{n}+\hat{c}_{n}^{\dagger}\right) / \sqrt{2}$ and $\hat{P}_{n}=i\left(\hat{c}_{n}^{\dagger}-\hat{c}_{n}\right) / \sqrt{2}$ and $\mathbf{X}=$ $\left(P_{0},-Q_{0}, P_{1},-Q_{1}, \ldots, P_{N-1},-Q_{N-1}\right)$. It is straightforward to show that, for a given density operator $\hat{\rho}, \chi_{c}$ is the same as the usual characteristic function, $\chi_{b}$, which is obtained in terms of modes $b_{m}: \chi_{c}(\mathbf{X})=\chi_{b}(\mathbf{x})$ where $\mathbf{x}$ is conjugate to $\mathbf{X}$ by Fourier transformation (3). The collective modes $c_{n}$ provide a different perspective from the modes $b_{m}$, preserving all physical properties for a given state.

The time-evolution operator $\hat{U}_{I}(\tau)$ for the interaction Hamiltonian (2) is equivalent to a beam splitter operator with the system mode $a_{2}$ and a collective mode $c_{0}$ as its input ports. That is,

$$
\hat{U}_{I}(\tau)=\exp \left[\theta(\tau)\left(\hat{a}_{2} \hat{c}_{0}^{\dagger}-\hat{a}_{2}^{\dagger} \hat{c}_{0}\right)\right],
$$

where $\theta(\tau)=\sqrt{N / 2} \int_{0}^{\tau} \lambda_{N}(t) d t$ determines the transmittivity, $t^{2}(\tau)=\cos ^{2} \theta(\tau)$. We take the limit, $N \rightarrow \infty$, keeping the transmitted energy finite, $t^{2}(\tau)=\exp (-\gamma \tau)$, in accordance with the Fokker-Planck equation. We find an important fact that the interaction of the system with the infinite modes $b_{m}$ of the environment can be reduced into the interaction with the single collective mode $c_{0}$. The properties of collective mode $c_{0}$ changes due to the interaction but each environmental mode $b_{m}$ hardly changes which is reflected in no change of other collective modes $c_{n}$.

A Gaussian field has the characteristic function in the form of $\chi(\mathbf{x})=\exp \left(-\mathbf{x} \mathbf{V} \mathbf{x}^{T} / 4\right)$ where $\mathbf{V}$ is the correlation matrix whose elements determine the mean quadrature values of the field: $V_{i j}=\left\langle\left(\hat{x}_{i} \hat{x}_{j}+\hat{x}_{j} \hat{x}_{i}\right)\right\rangle$. Note that we neglected linear displacement terms in the Gaussian characteristic function as they do not play a crucial role in the entanglement. The entanglement nature of a Gaussian field is thus uniquely represented by its correlation matrix $\mathbf{V}$. For a two-mode squeezed state, the correlation matrix $\mathbf{V}_{s}$ is simply [8]

$$
\mathbf{V}_{s}=\left(\begin{array}{cc}
\cosh (2 s) \mathbb{1} & \sinh (2 s) \boldsymbol{\sigma}_{z} \\
\sinh (2 s) \boldsymbol{\sigma}_{z} & \cosh (2 s) \mathbb{1}
\end{array}\right)
$$

where $\mathbb{1}$ is the $2 \times 2$ unit matrix and $\sigma_{z}$ is the Pauli matrix.

As the system interacts only with the collective mode $c_{0}$ in the homogeneous thermal environment, it suffices to consider the correlation matrix of the two system modes $a_{1}$ and $a_{2}$ and the collective mode $c_{0}$. The collective mode $c_{0}$ is initially in a thermal state with the average number $\bar{n}$ of the collective bosons. Thus, the correlation matrix of $a_{1}, a_{2}$, and $c_{0}$ before the interaction is given by $\mathbf{V}_{0}=\mathbf{V}_{s} \oplus \tilde{n} \mathbb{1}$. The evolution operator $\hat{U}_{I}(\tau)$ is now described by the matrix

$$
\mathbf{U}_{I}=\left(\begin{array}{ccc}
\mathbb{1} & 0 & 0 \\
0 & t \mathbb{1} & -r \mathbb{1} \\
0 & r \mathbb{1} & t \mathbb{1}
\end{array}\right)
$$

where $r^{2}=1-t^{2}$. Then the correlation matrix for the system and environment after the interaction is obtained as $\mathbf{V}_{c}=\mathbf{U}_{I} \mathbf{V}_{0} \mathbf{U}_{I}^{T}$.

A separability condition was derived by Simon [10] that a two-mode Gaussian state is separable if and only if the partially momentum-reversed correlation matrix (or equivalently the partially transposed density operator) satisfies the uncertainty principle. The condition was extended to a biseparability condition between a single mode and a group of $N$ modes by Werner and Wolf [11, which reads that a Gaussian field of $1 \times N$ modes is biseparable if and only if

$$
\boldsymbol{\Lambda} \mathbf{V} \boldsymbol{\Lambda}-\frac{1}{2} \boldsymbol{\sigma}_{y}^{\oplus(N+1)} \geq 0,
$$

where $\boldsymbol{\Lambda}$ is a partial momentum-reversal matrix, $\mathbf{V}$ is the correlation matrix of $1 \times N$ modes and $\boldsymbol{\sigma}_{y}$ is the Pauli matrix. Here, $\boldsymbol{\sigma}_{y}^{\oplus(N+1)} \equiv \boldsymbol{\sigma}_{y} \oplus \boldsymbol{\sigma}_{y} \oplus \ldots \oplus \boldsymbol{\sigma}_{y}$.

We start with a short discussion on the dynamics of the entanglement for the system. In order to consider quantum statistical properties of the field of modes $a_{1}$ and $a_{2}$, we trace the total density operator over all environmental modes, which is equivalent to considering the correlation matrix $\mathbf{V}_{c}$ only for the modes $a_{1}$ and $a_{2}$ :

$$
\mathbf{V}_{c}\left(a_{1}, a_{2}\right)=\left(\begin{array}{cc}
\cosh (2 s) \mathbb{1} & t \sinh (2 s) \boldsymbol{\sigma}_{z} \\
t \sinh (2 s) \boldsymbol{\sigma}_{z} & \left(t^{2} \cosh 2 s+r^{2} \tilde{n}\right) \mathbb{1}
\end{array}\right) .
$$


It has to be emphasized that this correlation matrix is exactly the same as the solution of the Fokker-Planck equation (11). Using Simon's criterion in Eq. (8) [8, 10, the field of the modes $a_{1}$ and $a_{2}$ is separable when the transmittivity of the beam splitter is $t^{2} \leq \frac{\bar{n}}{1+\bar{n}}$ for the squeezing parameter $s \neq 0$. Note that the separability condition does not depend on the initial entanglement of the system as far as there is any entanglement in the initial instance. The separability of the two-mode squeezed state depends only on the temperature of the environment and the overall transmittivity of the beam splitters.

We now study the entanglement of the system and the environment. Here, instead of the entanglement of the system with an individual mode $b_{m}$ of the environment, we are interested in the biseparability of a system mode and the collection of the environmental modes. Let us first consider the entanglement of the modes $a_{1}$ and $c_{0}$. The correlation matrix $\mathbf{V}_{c}\left(a_{1}, c_{0}\right)$ is equivalent to $\mathbf{V}_{c}\left(a_{1}, a_{2}\right)$ in Eq. (9) if $r$ and $t$ are interchanged. The separability condition is found to be $t^{2} \geq \frac{1}{1+\bar{n}}$, which is again independent from the initial entanglement of the system as far as $s \neq 0$.

The entanglement of modes $a_{2}$ and $c_{0}$ is easily discussed using a quasiprobability $P$ function 叫, the existence of which is a sufficient condition for entanglement [12]. Tracing over mode $a_{1}$ of the two-mode squeezed state, the other mode $a_{2}$ is in a thermal state with $\tilde{n}_{s} \equiv 2 \bar{n}_{s}+1=\cosh 2 s$. It is well-known that thermal states have positive definite $P$ functions and the action of a beam splitter transforms only the coordinates of the input $P$ functions: $P_{a_{2}}(\alpha) P_{c_{0}}(\beta) \stackrel{b s}{\longrightarrow} P_{a_{2}}(t \alpha-$ $r \beta) P_{c_{0}}(t \beta+r \alpha)$. Thus the field of modes $a_{2}$ and $c_{0}$ is always separable. A simple decoherence picture, that a system decoheres as it entangles with its environment, does not hold any longer. However this does not mean that there is no entanglement involved in the decoherence process because the other mode of the system has to be taken into account.

Fig. 1 presents the entanglement structure for a twomode squeezed state interacting with a thermal environment. The solid lines are the boundaries of entanglement of the system mode $a_{1}$ and the collective mode of the environment $c_{0}$ and of the two system modes $a_{1}$ and $a_{2}$. These lines are obtained by the separability condition (8) in the present exactly solvable model. For the comparison, we consider $N=100$ beam splitters modeling the interaction with the thermal environment and calculate the biseparability of the $1 \times 100$-mode field using Giedke et al.'s computational analysis [13]. The computational results of entanglement are denoted by circles and dots. We find that the results are also independent of the squeezing parameter. Fig. 1 1 shows that the two methods are exactly consistent.

Now, we need to consider what Fig. 11 really tells us. The three modes $a_{1}, a_{2}$, and $c_{0}$ compose a tripartite system. Multi-mode entanglement for a continuous-variable system has been studied extensively using beam splitters and single-mode squeezed states [14]. Giedke et al. clas-

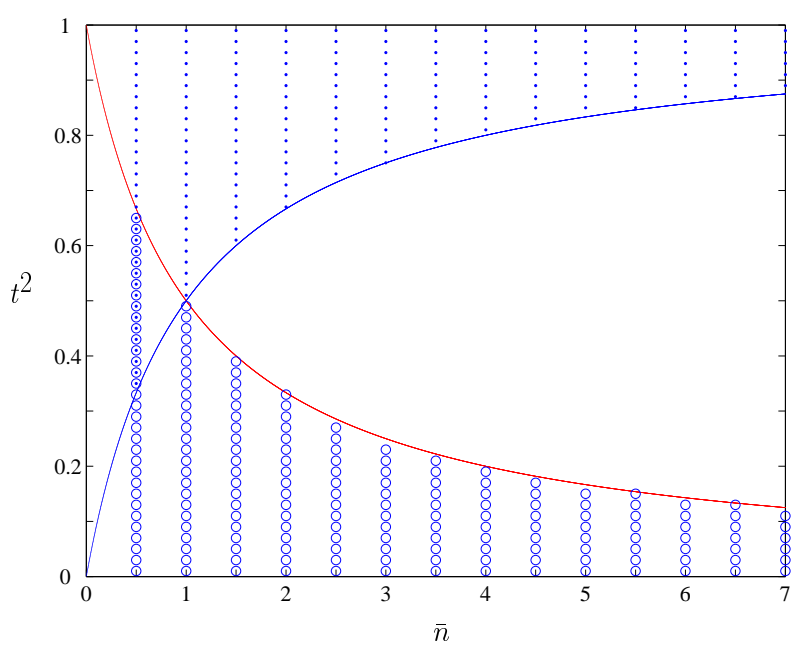

FIG. 1: Nature of entanglement for a two-mode squeezed state interacting with a thermal environment of the average photon number $\bar{n}$. $t^{2}=\exp (-\gamma \tau)$ is the transmittivity of the collective beam splitter. The solid lines are the boundaries of entanglement of $a_{1}$ and $c_{0}$ and of $a_{1}$ and $a_{2}$, which are obtained by the separability condition. The circles and dots are found by a computational analysis with $N=100$ beam splitters. The circles indicate that the system mode $a_{1}$ and the group of environmental modes $b_{m}$ are entangled and the dots indicate that the system modes $a_{1}$ and $a_{2}$ are entangled.

sified types of entanglement for a three-mode Gaussian field [13]. When a three-mode field is not biseparable, it is called fully entangled. Here, a three-mode field is biseparable when any grouping of three modes into two are separable. A fully-entangled tripartite qubit system may be entangled in two inequivalent ways [15, 16], one of which is Greenberg-Horne-Zeilinger (GHZ) entanglement [17] and the other is W-entanglement [15]. A GHZentangled state becomes separable when any one particle is traced over any one particle while a $\mathrm{W}$-entangled state is pairwise entangled for any pair of the three particles. On the other hand, there is another kind of entanglement for a fully-entangled tripartite $d$-dimensional system [18]. That is, two-way entanglement: One example for three particles labeled as $a, b$ and $c$ are pairwise entangled of $a$ and $b$ and of $b$ and $c$ but the particles $a$ and $c$ are separable.

From the biseparability condition in Eq. (8), we find that the tripartite system of $a_{1}, a_{2}$, and $c_{0}$ is fully entangled if $0<t^{2}<1$ and $s \neq 0$. It is notable that the tripartite system being fully entangled is independent of the temperature of the environment and thus over whole region in the Fig. 1. In Fig. 1, it is shown that the tripartite system is two-way entangled, i.e., $a_{1}-a_{2}$ and $a_{1}-c_{0}$ modes are entangled for $\bar{n}<1$ if $\frac{\bar{n}}{1+\bar{n}}<t^{2}<\frac{1}{1+\bar{n}}$. There are two regions where only one pair of the three-mode field is entangled. 
We focus on the region of $\bar{n}>1$ described by

$$
\frac{1}{1+\bar{n}} \leq t_{0}^{2} \leq \frac{\bar{n}}{1+\bar{n}}
$$

where there is no pairwise entanglement. Because the tripartite system of $a_{1}, a_{2}$ and $c_{0}$ are fully entangled, if there is no pairwise entanglement, the only possibility is GHZ-entanglement among the three modes. Here, we draw the conclusion that $G H Z$ entanglement of an entangled system with an environment is a source to its loss of the initial entanglement. This is clearly seen in Fig. 1 when we consider the dynamics of the entanglement as the interaction time $\tau$ increases or equivalently the transmittivity $t^{2}$ decreases. For $\bar{n}>1$, the two modes of the system are initially entangled. As they come to the region where they are GHZ-entangled with the environment, the two modes of the system lose their initial entanglement. Finally, the entanglement is transferred to between the system mode $a_{1}$ and the collection of the environmental modes.

When system mode $a_{2}$ is neither GHZ-entangled nor pairwise entangled with $a_{1}$, what happens to $a_{2}$ ? In order to understand it, let us consider each thermal mode of the environment as a part of a pure system. Then the total system comes to be in a pure state, which is easier to analyze. As pointed out earlier, by tracing over one mode of a two-mode squeezed state, we have a single-mode thermal field with $\tilde{n}=\cosh 2 s_{n}$. We may consider a purification of the thermal environmental state such that the thermal mode $b_{m}$ results from tracing a pure two-mode squeezed state $\hat{\rho}_{b_{m} b_{m}^{\prime}}$ over its counter mode $b_{m}^{\prime}$. Letting $c_{0}^{\prime}$ be the counter collective mode of $c_{0}$, the modes of $a_{1}, a_{2}, c_{0}$ and $c_{0}^{\prime}$, that involve in the interaction, compose the pure total system. As the system mode $a_{2}$ interacts with the collective mode $c_{0}$, there should be an analogy between the relations of the modes $a_{1}, a_{2}$, and $c_{0}$ and those of the modes $c_{0}^{\prime}, c_{0}$, and $a_{2}$. We have already seen that modes $a_{1}$ and $c_{0}$, which have never met, may get entangled by the long interaction. This argument can be applied to the entanglement of the modes $c_{0}^{\prime}$ and $a_{2}$. Here, we obtain an interesting result that the system mode $a_{2}$, which was initially entangled with the system mode $a_{1}$, loses its initial entanglement and becomes entangled not with the interacting environmental modes but with the hidden environmental modes, $c_{0}^{\prime}$. The entanglement of $c_{0}$ and $c_{0}^{\prime}$ can be lost but the entanglement of $b_{m}$ and $b_{m}^{\prime}$ is almost kept unchanged as other collective modes $c_{n}$ and $c_{n}^{\prime}$ are still well-entangled. For the comparison with the present model, we calculate the separability of the system mode $a_{2}$ and the group of the hidden modes $b_{m}^{\prime}$ by employing 100 beam splitters and using Giedke et al.'s computational analysis. This results in the same as the dots in Fig. 1 with $\bar{n}$ of the $x$-axis replaced by $\left(\tilde{n}_{s}-1\right) / 2$. On the other hand, if $\bar{n}$ of the environment is zero, the environment is in a pure vacuum state and there is no hidden environmental modes. In this case, the system of $a_{1}$ and $a_{2}$ becomes never separable.

In this paper, we have studied the decoherence mechanism by highlighting the entanglement of the continuousvariable system with its environment. We showed that the homogeneous thermal environment can be summarized by a single collective mode with respect to the interaction with the system for the study of entanglement. As the two-mode entangled system is interacting with the thermal environment, the two modes may lose their entanglement by the GHZ-entanglement with the group of the environmental modes. The initial entanglement of the two-mode system transfers to the entanglement of one of the system modes and the group of environmental modes. We also studied the nature of entanglement for the other system mode.

\section{Acknowledgments}

We thank Prof. V. Bužek for discussions.
[1] W. H. Zurek, Phys. Today, p.31 (Oct. 1991).

[2] W. H. Zurek et al., Phys. Rev. Lett. 70, 1187 (1993).

[3] Some exceptions can be found. See, for example, J.H. Reina et al., Phys. Rev. A 65, 032326 (2002); M. S. Kim, J. Lee, D. Ahn and P. L. Knight, Phys. Rev. A 65, 040101 (R) (2002).

[4] S. M. Barnett and P. M. Radmore, "Methods in Theoretical Quantum Optics," (Clarendon Press, Oxford, 1997).

[5] M. S. Kim and N. Imoto, Phys. Rev. A 52, 2401 (1995).

[6] D. Nagaj, P. Stelmachovic, V. Buzek, and M. S. Kim, Phys. Rev. A (in print, 2002).

[7] A. Furusawa et al., Science 282, 706 (1998).

[8] M. S. Kim, J. Lee and W. J. Munro, Phys. Rev. A 66, 030301 (R) (2002).

[9] S. L. Braunstein and J. Kimble, Phys. Rev. Lett. 80, 869 (1998); Č. Brukner et al., e-print quant-ph/0208116 (2002).
[10] R. Simon, Phys. Rev. Lett. 83, 2726 (2000).

[11] R. F. Werner and M. M. Wolf, Phys. Rev. Lett. 86, 3658 (2001).

[12] J. Lee, M. S. Kim and H. Jeong, Phys. Rev. A 62, 032305 (2000).

[13] G. Giedke et al., Phys. Rev. Lett. 87, 167904 (2001); G. Giedke et al., Phys. Rev. A 64, 052303 (2001).

[14] P. van Loock and S. L. Braunstein, Phys. Rev. Lett. 84, 3482 (2000); P. van Loock and S. L. Braunstein, Phys. Rev. A 63, 022106 (2001).

[15] W. Dür et al., Phys. Rev. A 62, 062314 (2000).

[16] A. Acín et al., Phys. Rev. Lett. 87, 040401 (2001).

[17] D. M. Greenberger et al., Bell's Theorem, Quantum Theory, and Conceptions of the Universe, edited by M. Kafatos (Kluwer, Cordrecht, 1989).

[18] A. V. Thapliyal, Phys. Rev. A 59, 3336 (1999). 